\title{
COVID-19 pandemic management at the Emergency Department: the changing scenario at the University Hospital of Pisa
}

\author{
Greta Barbieri, ${ }^{1}$ Stefano Spinelli, ${ }^{2}$ Matteo Filippi, ${ }^{3}$ Francesca Foltran, ${ }^{3}$ Mauro Giraldi, ${ }^{3}$ \\ Maria Carola Martino, ${ }^{3}$ Alessandro Cipriano, ${ }^{2}$ Francesco Cinotti, ${ }^{2}$ Massimo Santini, ${ }^{2}$ \\ Lorenzo Ghiadoni ${ }^{4}$ \\ ${ }^{1}$ Department of Surgical, Medical, Molecular and Critical Area Pathology, University of Pisa; ${ }^{2}$ Emergency \\ Medicine Department, University Hospital of Pisa; ${ }^{3}$ Medical Directional Department, University Hospital of \\ Pisa; ${ }^{4}$ Department of Clinical and Experimental Medicine, University of Pisa, Pisa, Italy
}

\begin{abstract}
Starting from $1^{\text {st }}$ case in Italy, on February 20th, 2020, CO-rona VI-rus D-isease 2019 (COVID-19) pandemic spread to whole Italian territory, with different regional distribution. Tuscany has been classified as medium diffusion area (40-100 cases/100000 inhabitants). In this context, all healthcare structures reviewed their organization to meet new needs. Our study's objectives were description of organizational model outlined to safely manage Emergency Department (ED) and analysis of patients' flows within Hospital of Pisa during pandemic. The ED has been reorganized with dedicated areas for examination and waiting for tests results. A similar reduction (-62\%) of ED accesses comparing to the same period of 2019 and the previous months of 2020 was observed. Hospital Task Force arranged for progressive activation of Units by modules, according to territorial needs. From the beginning of March to the end of April 2020, 315 COVID-19 patients were hospitalized. Overall, a $45 \%$ reduction in hospital admissions compared to the same period of 2019 was observed, with increased mortality (4\% versus $2 \%$ ). The University Hospital of Pisa efficiently managed COVID-19 emergency with a logistical reorganization of ED.
\end{abstract}

\footnotetext{
Greta Barbieri, Department of Surgical, Medical, Molecular and Critical Area Pathology, University of Pisa, via Savi, 10, 56126 Pisa, Italy

Tel.: +39-3470104897

E-mail: greta.barbieri@phd.unipi.it

Key words: Pandemic; Covid-19; Management; Emergency.

Conflict of Interest: the authors declare no potential conflict of interests. Ethics approval and consent to participate: The manuscript does not contain any individual person's data in any form.

Received for publication: 29 May 2020.

Accepted for publication: 2 July 2020

This work is licensed under a Creative Commons Attribution 4.0 License (by-nc 4.0).

C. Copyright: the Author(s), 2020

Licensee PAGEPress, Italy

Emergency Care Journal 2020; 16:9146

doi:10.4081/ecj.2020.9146
}

\section{Introduction}

The World Health Organization (WHO) declared the coronavirus 2 (SARS-CoV-2) infection outbreak a pandemic due to the constantly increasing number of cases outside China after the first one found at Wuhan. ${ }^{1,2}$ The CO-rona VI-rus D-isease identified in 2019 (COVID-19) is linked to SARS-CoV-2 transmission via droplets and it is characterized by a spectrum of clinical manifestations from flu-like symptoms to a Severe Acute Respiratory Syndrome (SARI). ${ }^{3}$ The first diagnosed case in the Italian territory was reported on the February 20th $2020 .^{2}$

Thereafter, the Italian COVID-19 epidemic has been mainly characterized by a local transmission. Accordingly, several preventive measures to favor social distancing were undertaken, initially at the local level and finally at the national level with a lockdown of the entire territory on March 11th 2020 because of the increasing positive cases. ${ }^{2,4,5}$

A rapid diffusion of SARS-CoV-2 positive cases, along with the increase of accesses to Emergency Department (ED) for COVID-19 and the number deaths occurred, particularly in the northern regions and thereafter in the rest of Italy. ${ }^{6,7}$ A report produced jointly by Italian Institute of Statistics (ISTAT) and National Institute of Health (ISS) identified heterogeneous geographical spread of the COVID-19 epidemic. ${ }^{8}$ The distribution of standardized cumulated incidence rates from March 5th to 31st of cases confirmed positive for the infection allowed to identify 3 classes: low diffusion (lower than 40 cases per 100000 residents), medium (between 40 and 100 cases per 100000 residents), high diffusion, (values above 100 cases per 100000 residents). In medium- and low- incidence areas, the number of cases began to increase from mid-March reaching a peak, respectively, between March 24th and 25 th $2020 .^{8}$

Although local and national preventive measures started since the end of February, following the update on epidemiological criteria, the curves of diagnosed cases and deaths continued to grow until the last week of March 2020. ${ }^{8,9}$

Accordingly, documents from national and regional authorities were continuously updated, focusing also on the hospital's organization facing the pandemic outbreak. ${ }^{10}$ On March $14^{\text {th }}$ Guidelines for management of COVID-19 patients in Tuscany by expert panel (including MS and LG) were released. ${ }^{11}$

On February $24^{\text {th }}$, Hospital Task Force for COVID-19 emergency management was activated at University Hospital of Pisa.

In this manuscript, we describe COVID-19 outbreak management in a tertiary-care, the University Hospital of Pisa, Italy. 


\section{Materials and Methods}

The University Hospital of Pisa, Italy is one of the three tertiary-care hospitals of Tuscany, serving as local nosocomial center for the city of Pisa (approximately 100.000 inhabitants) and referral center for the North-West part of the region (approximately 1 million 200 thousand inhabitants). The present analysis has the objectives description of ED rearrangement during COVID-19 emergency and evaluation: i) Patient flows in ED during COVID19 emergency; ii) Hospitalization during COVID-19 emergency; iii) COVID-19-related mortality. We analyzed retrospectively the accesses to ED of University Hospital of Pisa in March 2020, by a comparison between a period prior to COVID-19 emergency and a similar duration period of 2019, in terms of: i) Absolute Number; ii) Territorial origin; iii) Hospitalization. Moreover, we recorded the timing activation of COVID-19-dedicated Departments, responding to specific territorial needs, according the Guidelines for management of COVID-19 patients in hospital in Tuscany. ${ }^{11}$

Finally, we evaluated within hospitalized positive-COVID19 patients' group: age of population; hospitalization setting (ordinary stay or intensive care); length of hospital stays. The period considered in ED access analysis was from March 1st to March 31st. As regards to hospitalization analysis, April was also included. Data was extracted from FirstAid $\AA$, electronic medical record program used in ED and from the Production Sector database of University Hospital of Pisa.

The Internal Review Board (IRB) approved the study (number 230320). Patients with pneumonia and laboratory confirmed COVID-19, diagnosed by a positive SARS-CoV-2 RT-PCR test on a Nose-Pharyngeal Swab (NPS), were included in the study.

\section{Results}

\section{Emergency Department}

The diagram in Figure 1 shows patient's diagnostic flow chart within the University Hospital of Pisa ED.

All Hospital accesses have been closed except for strategic points identified as checkpoints, dedicated to identification (employee or patient or visitor), after hands sanitation and facial protective mask delivery.

A pre-triage outpost, consisting of a pneumatic tent suitably set up and positioned in front of the ED entry was identified as Pretriage tent. Multilingual info-graphic material (Italian/English/ Chinese) and specific paths have been developed for identifying patient with SARS-CoV-2 infection case clinical-epidemiological criteria with spontaneous presentation in ED and adopting immediately transmission prevention procedures.

Furthermore, the Territorial Emergency Central operator contacted the ED triage by notifying of a COVID-19 possible suspect case arrival.

Criteria used for defining cases as suspicion for COVID-19 infection were: i) Acute respiratory infection, in the absence of an alternative diagnosis and positive history for travel or residence in areas of high contagion; ii) Acute respiratory infection having contact with probable or confirmed cases of COVID-19 in the previous 14 days; iii) Severe acute respiratory infection requiring hospitalization, in absence of alternative cause.

Subjects with COVID-19 infection suspicion criteria identified in pre-triage and triage, were treated in dedicated areas (COVIDarea $E D$ ) by dedicated healthcare staff (other than no-COVD area working group), wearing the recommended Personal Protective Equipment (PPE, such as masks, goggles, clothes and gloves). Three ED dedicated modules were identified (maximum capacity of 30 patients), and negative pressure zone were set up to assist safely patients needing ventilation or aerosolization generating procedures. Patients (both walking and stretched) entered the COVID area through dedicated dirty paths, which never crossed the no-COVID area.

Access to ED Radiology was regulated by dedicated staffs to flows management, coordinated with a cleaning and sanitizing service activated for 24 hours a day, for guaranteeing immediate decontamination of promiscuous passage zones.

Moreover, a specific area has been identified for patients waiting for NPS, performed in all subjects requiring hospitalization, despite access not attributable to COVID-19 symptoms.

In order to reduce waiting times, dedicated personnel carried out transport service of NPS, with appropriate hygienic precautions regarding the sample packaging.

The virology staff was implemented with new hires and was effective 24 hours a day leading to an average waiting times for NPS result were 6-8 hours.

A new informatic application was created for viewing the samples processing and real-time reporting. Therefore, in case of overcrowding and saturation of maximum places in the dedicated area to the evaluation of suspect cases, patients were directly admitted to the COVID area of ordinary hospital stay, without waiting for the results of the NPS test (Figure 1, orange area).

Patient evaluation provides, according with Regional Guidelines, ${ }^{11}$ the following test:

i) Vital signs (blood pressure, heart rate, temperature, clinical deterioration scale); ii) Blood gas analysis (including arterial oxygen partial pressure/ fractional inspired oxygen ratio, $\mathrm{P} / \mathrm{F}$ ); iii) Respiratory clinic evaluation (dyspnea degree, walking test); iv) Basic laboratory tests profile (including cell blood count, hepatic, renal, inflammation indices, high sensitive troponin); v) Thoracic imaging (Lung Ultrasound, LUS and chest Computed Tomography, CT); vi) NPS.

Test results allow patient's classification into disease levels, necessary for subsequent management. ${ }^{11}$ In particular, 4 stages of clinical severity were identified, which corresponded to different care settings: i) Level 1: Patients with non-specific symptoms, absence of hypoxia (Oxygen saturation $>94 \%$ ), negative imaging. This category could be sent home, delegating its management to the territorial services; ii) Level 2: Patients with fever, respiratory failure, respiratory rate $(\mathrm{RR})>25$ acts/minute, $\mathrm{P} / \mathrm{F}>200$, positive imaging for pneumonia. These subjects must be hospitalized in ordinary hospital stay with oxygen therapy and monitoring; iii) Level 3: Patients with fever, respiratory failure, RR $>30$ acts / minute, P/F 100-200, positive imaging for pneumonia. Hospitalization in the sub-intensive area is suggested; iv) Level 4: Patients with severe respiratory failure, respiratory rate or Acute Respiratory Distress syndrome features and high probability of invasive support, positive imaging for pneumonia. Hospitalization in an intensive area is necessary.

Regarding imaging, ED Radiology was able to perform baseline chest CT in 70\% of COVID-19 positive patients before hospitalization, by dedicated CT scan.

Furthermore, LUS allowed evaluating patients in all care settings and in all phases of hospitalization, being performed in more than $60 \%$ of COVID-19 hospitalized patients.

Specific pathways were delineated for all time dependent pathologies (stroke, acute coronary syndrome, trauma) to be implemented in suspected COVID patients. 
A COVID Bedding Monitoring and Management System (called VISUAL COVID) has been set up, allowing the real-time monitoring of cases waiting at ED and the availability of hospitalization in different intensity care areas. This service was managed by Medical Directional Department staff, whose task was to sort patients from emergency room to the appropriate care setting identified by ED assessment, complemented by resuscitation counsel when necessary.

In March 2020, a significant decrease of patients' flow in ED as compared to the previous months (106 accesses/daily in March versus 243 in January, 62\% reduction), as shown in Figure 2.

A $59 \%$ reduction in total accesses to ED was observed in March 2020 as compared to the same month of previous year (2626 versus 6334). The total number of accesses in the month of April was 2132 , compared to 6237 in the previous year $(66 \%$ reduction).

Figure 3 shows the global accesses in March and April. It can be observed a reduction in total access to ED, greater starting from 04 March, corresponding to the first positive COVID-19 case in Pisa. The number of patients discharged at home is significantly higher than hospitalization in the first days of the month. Instead, after March 10, the 2 groups are numerically similar. A low mortality rate in the early stages of patient management was also observed.

\section{Hospitalization}

A dedicated area (named COVID Bubble) has been identified, in order to ensure correct management of patients with infection by COVID-19, with sub-sequent involvement of different hospitals' Departments, including one Infectious Diseases, one Pneumology Unit, four Internal Medicine Units and one Resuscitation Unit.

The staff of these Units has been reorganized, guaranteeing in each group a resuscitating physician, an infectious disease specialist and a thoracic sonographer, as stable consultants for each shift. In addition, the specialists were redistributed in all the COVID-19 Units, to assist teams in covering shifts and in managing specialist complications that occurred in hospitalized COVID-19 patients.

From March $1^{\text {st }}$ to April $30^{\text {th }} 2020,315$ patients with SARSCov2 pneumonia were overall hospitalized in our Hospital. The average daily presence of COVID 19 positive patients has gradually increased from March 16th, reaching the maximum level of 187 on March $28^{\text {th }}$.

Patients admitted to the COVID-Hospital had a higher average age than the age recorded in the whole Hospital (69 years compared to 58 years) and a higher average hospital length-of-stay (12 days compared to 6 days).

We observed a significant percentage reduction in hospitalizations during March and April 2020, compared to the previous year, regarding all types of admissions (globally 45\%). However, we observed an increase in mortality: 188 patients out of 4582 (equal to $4,1 \%$ of the total) compared to 205 out of 9946 , equal to $2,06 \%$ of the total (Figure 4).

The departments dedicated to COVID-19 patients have been activated progressively, based on the specific needs of the moment, reaching a total of 184 ordinary beds and about 58 sub-intensive and intensive beds. We recorded a peak of admissions from $3 / 20 / 20$ to $3 / 31 / 20$, characterized by temporary patients overflow from areas outside of Pisa, in correspondence with the closure of peripheral hospitals in the North-West area of Tuscany (Figure 5)

\section{Discussion}

The EDs faced an arduous organizational challenge during COVID-19 pandemic. Indeed, it was mandatory to guarantee the safety of patients and health workers with adequate paths, even managing a large number of suspect patients. In the case of patient with $S A R S-C o V-2$ infection case clinical-epidemiological criteria, early identification is essential, to immediately adopt the transmission prevention procedures, reducing possible contacts. ${ }^{12}$

Our hospital has set up dedicated areas for examining and waiting for diagnostic tests for patients with suspicious symptoms, in addition to specific pathways for access to laboratory and instrumental services, in order to maintain operator safety and system efficiency.

Diagnosis represents a limiting and difficult stage for COVID19 patient's management, due to NPS low sensitivity. ${ }^{13}$ Furthermore, long term stays in ED were very problematic, particularly for patients with suggestive symptoms but first negative NPS and during overcrowding phases. For this reason, imaging tests played a significant role, allowing for rapid risk stratification in suspected symptomatic patients in ED. ${ }^{14}$ This approach was possible thank to the availability of ED Radiology to perform a large number of baseline chest CT before hospitalization and consolidat-

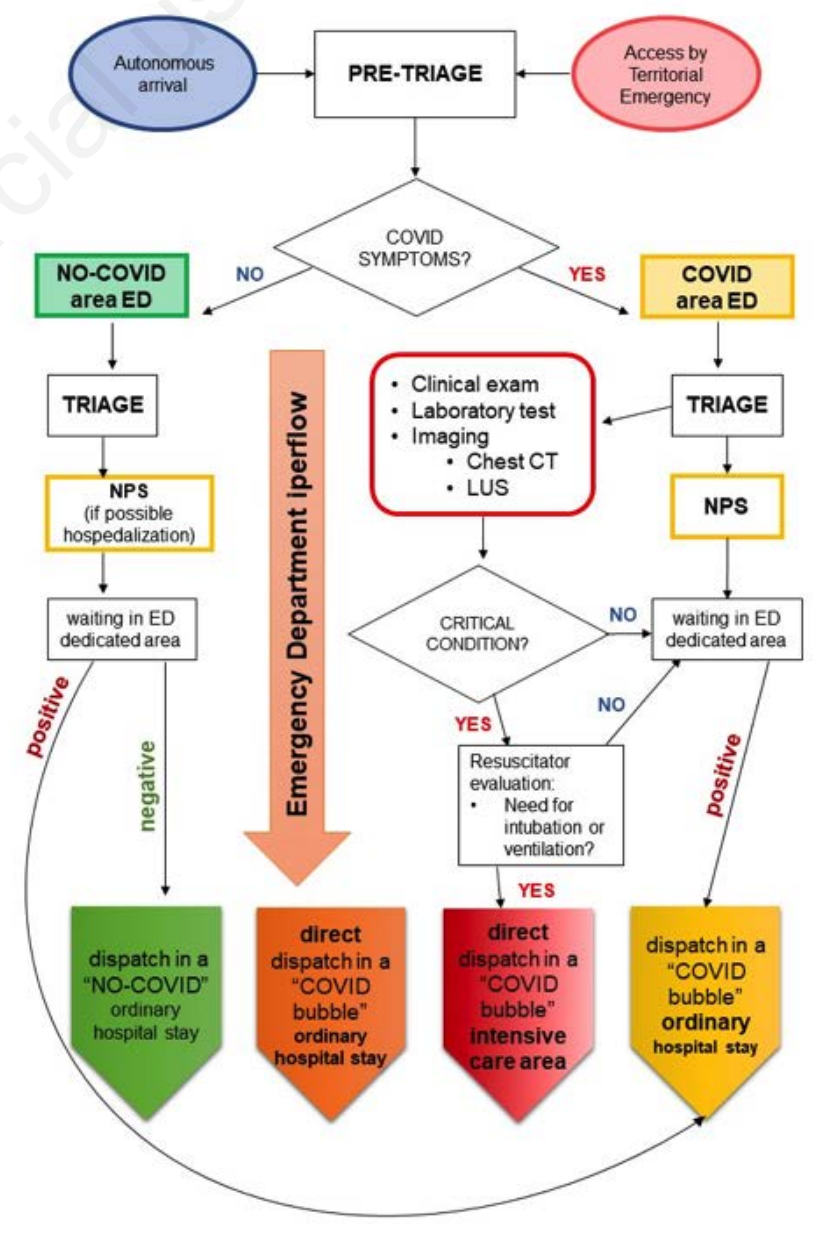

Figure 1. Emergency Department COVID-suspected patients flow chart. ED: Emergency Department, NPS: nose-pharyngeal swab, LUS: lung ultrasound, CT: computed tomography. 
ed experience on LUS, which allowed to evaluate patients in all care settings and in different phases of hospitalization, with standardized technique and documented report. ${ }^{15}$

Our descriptive analysis shows a dramatic reduction of patients' flow in ED during COVID-19 pandemic in University Hospital of Pisa compared to the previous months. This is a reliable consequence of the population's fear on possible contact with people positive for Sars-COV-2, avoiding access to ED. Several considerations should be addressed from this evidence. Firstly, life-threating diseases, particularly time-depending such as acute coronary syndrome and stroke, would have been unrevealed and untreated. On the other hand, acute situations such as trauma have been expected to be almost null. Nevertheless, it is conceivable that the number of access in pre-COVID period were consistently inappropriate causing crowing and boarding in the ED. ${ }^{16}$

Secondly, these data show a substantial change in the use of ED by population, clearly reducing their presence in the Hospital. This raises the issue of improper use of the ED for diseases that are not really urgent.

Considering all accesses to ED in March and April 2020, we observed a significant reduction of patients discharged at home as compared to those requiring hospitalizations. This was related to the fewer accesses to ED from one hand and a possible the conservative management adopted in ED in SARS- CoV2 positive patients, who were detained for observation or investigations in most cases, on the other. Interestingly, we also observed a very low mortality in the early stages of patient's ED management. It is conceivable that the observed reduction in overall number of hospitalizations was due to for a significant portion those not admitted by ED (for example elective admissions in the first phases of pandemic).

Correct management and rapid adaptation of the hospitals is essential, as COVID-related deaths can be indirect causes to failure to maintain the health system. ${ }^{12}$

The geographical spread of the COVID-19 epidemic was heterogeneously distributed in Italy. In the Southern Regions and islands, the spread of infections was very limited, in the Center, it was on average higher, while in the North the circulation of the virus was very high. The curve of diagnosed cases slowed down due to the measures of lockdown undertaken throughout the

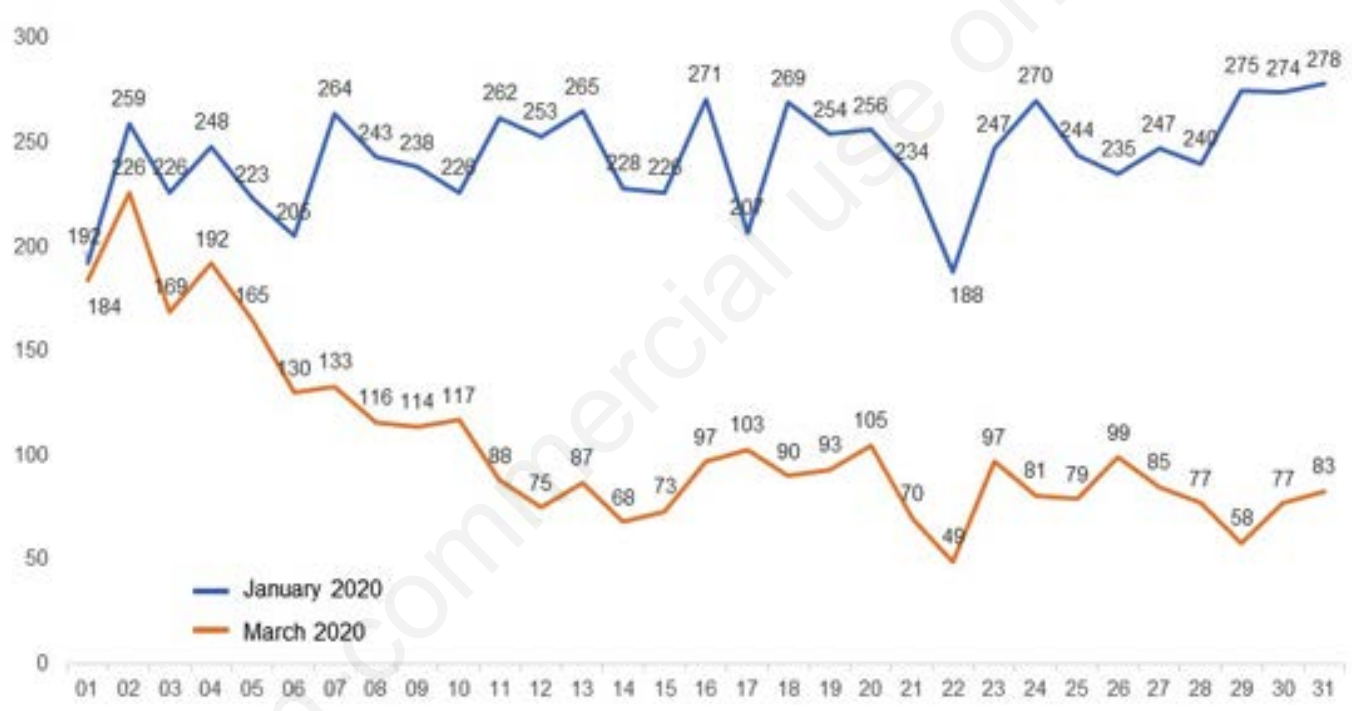

Figure 2. Emergency Department accesses on January 2020 compared to March 2020.

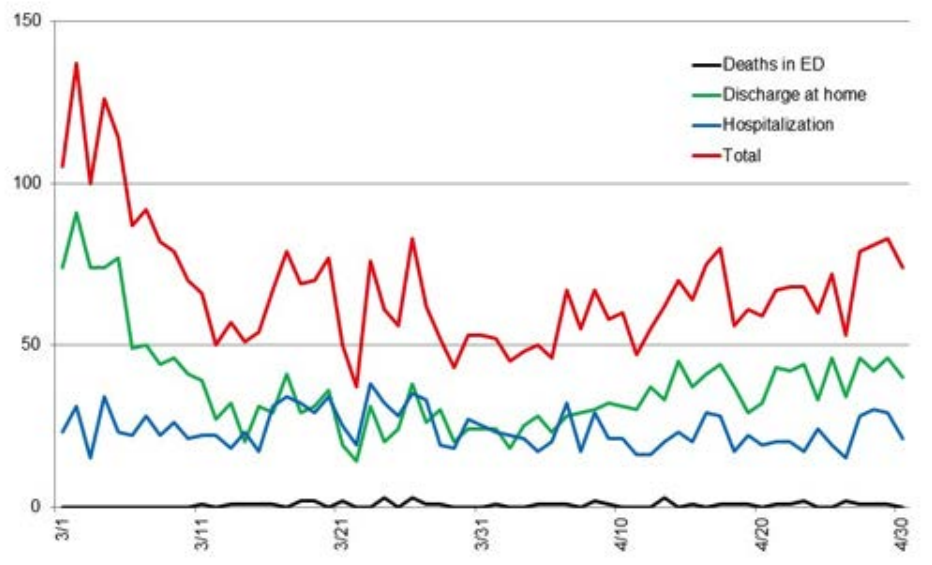

Figure 3. Emergency Department management of global accesses from 1st March to 30 April 2020. 
national territory since March $11^{\text {th }}$. In the specific case, our hospital had to cope with a great overflow from peripheral hospitals of Northwest zone of Tuscany. Indeed, these areas had to manage a very high number of COVID patients possibly caused by people originating from Lombardy movement to the coast in the $1^{\text {st }}$ weekend of March. ${ }^{17}$ Alternately, the management of Northwest Tuscany area hospitals did not react promptly to the increase flow of COVID-19 patients to their EDs. Our hospital managed COVID-19 emergency by activating coordination corpses for the constant monitoring of territory request and system response capacity. This allowed a rapid reorganization of the Departments by intensity of care and a prompt activation by modules of the various Units, in a time dependent method, corresponding to the periods of greatest territorial need.

Since 90's of the last century, an emergency plan for mass casualties is mandatory for all the Italian hospitals. ${ }^{18}$ This planning is related with several identified risk models, mainly focus on trauma cares (earthquakes, big accidents, terroristic attacks, flooding); the plans are hospital-based, but the Italian Civil Protection System provides supervision and an emergency network strategy of the National Health System NHS. ${ }^{19}$ After the 2015 Ebola epidemic, all Italian hospitals identified a biohazard emergency pathway. However, it does not cope with high number of patients admitted as it occurs during a pandemic. Facing the COVID-19 outbreak, just the really first cases were managed according with these plans, forcing the NHS to shift on a new coping strategy. ${ }^{12,20}$ The rapid modulation of hospital organization proved to be fundamental in COVID-19 emergency, in order to respond promptly to specific territorial needs in all areas affected by pandemic..$^{20}$
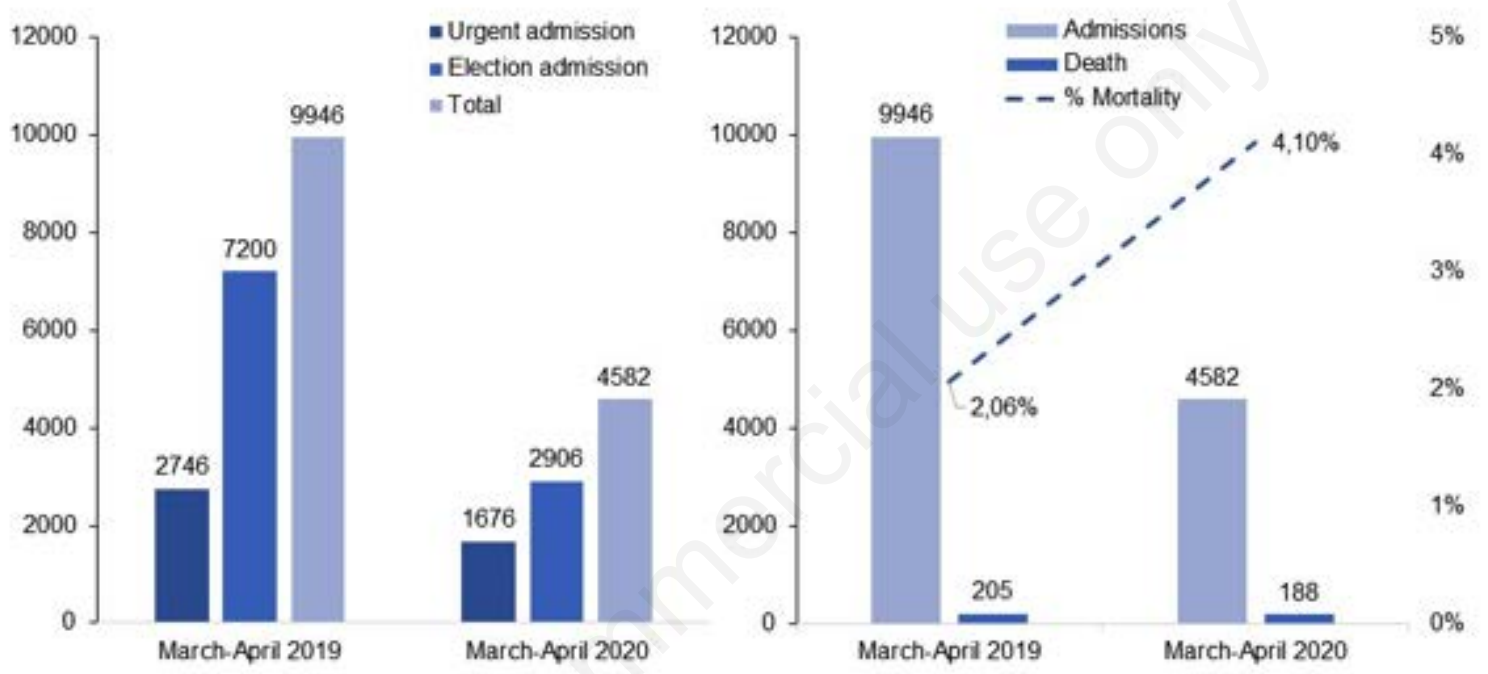

Figure 4. Hospitalization in March-April 2019 compared to March-April 2020.

\begin{tabular}{|l|r|r|}
\hline Department & N. beds & Activation date \\
\hline Infectivous Disease COVID Unit & 23 & $03 / 06 / 2020$ \\
\hline Intensive Care COVD Unit & 46 & $03 / 11 / 2020$ \\
\hline Pneumology COVD Unit & 19 & $03 / 15 / 2020$ \\
\hline Internal Medicin COVD Unit & 36 & $03 / 19 / 2020$ \\
\hline Urgency Medicine COVD Unit & 36 & $03 / 22 / 2020$ \\
\hline Internal Medicin V COVD Unit & 36 & $03 / 24 / 2020$ \\
\hline University Geriatrics COVD Unit & 34 & $03 / 27 / 2020$ \\
\hline
\end{tabular}

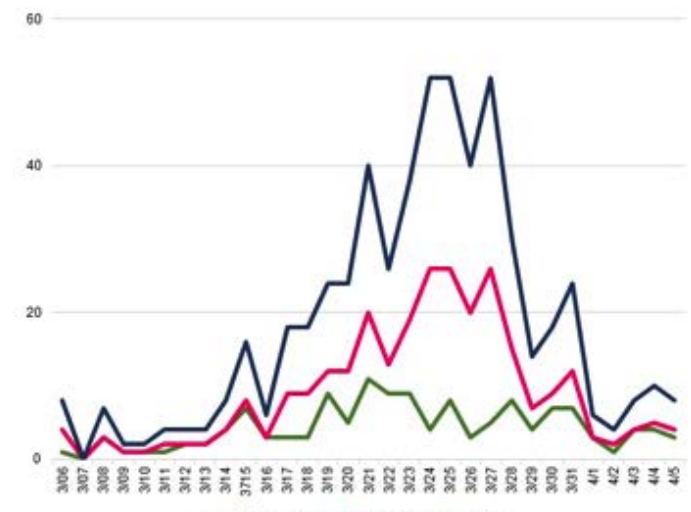

Figure 5. Activation phases of University Hospital of Pisa resources and average daily presence of COVID-19 patients from $10 / 03 / 2020$ to $10 / 04 / 2020$, including those from Pisa and Northwest Tuscany area. 


\section{Conclusions}

In our Hospital, the higher access load was managed using a module activation model of multiple operating units, based on local requirements. Patients were organized by intensity of care and treated by multidisciplinary teams.

Facing this new and unexpected wide pandemic a failure of hospital's emergency plan was observed. However, a rapid cope has been achieved through smart solutions and thanks to a good baseline logistic. We would like to advocate for that lesson will move to a more comprehensive pandemic emergency plan, locally and national-wide.

In a subsequent post-COVID phase, a conversion of Territorial Emergency system and hospital ED will be required with the aim of avoiding crowding and boarding. The changing scenario of ED will be assessed nationally by a targeted AcEMC study.

\section{References}

1. World Health Organization. General's opening remarks at the media briefing on COVID-19:11 March2020. 2020. Available from: https://www.who.int/dg/speeches/detail/who-directorgeneral-s-opening-remarks-at-the-media-briefing-on-covid19---20-march-2020

2. ArcGIS. Coronavirus COVID-19 global cases by the Center for Systems Science and Engineering (CSSE) at Johns Hopkins University (JHU). 2020. Available from: https://gisanddata.maps.arcgis.com/apps/opsdashboard/index. html\#/bda7594740fd40299423467b48e9ecf6

3. Huang C, Wang Y, Li X, et al. Clinical features of patients infected with 2019 novel coronavirus in Wuhan, China. Lancet 2020;395:497-506.

4. Italian Ministry of Health. Covid-19. [Situazione in Italia. 2020.] Available from: http://www.salute.gov.it/portale/nuovocoronavirus/dettaglioContenutiNuovoCoronavirus.jsp?lingua $=i t$ aliano $\&$ id $=5351 \&$ area $=$ nuovoCoronavirus $\&$ menu $=$ vuoto

5. Italian Ministry of Health. [Decreto \#IoRestoaCasa, domande frequenti sulle misure adottate dal Governo]. Available from: http://www.governo.it/it/faq-iorestoacasa [Website in Italian].

6. Grasselli G, Zangrillo A, Zanella A, et al. Baseline Characteristics and Outcomes of 1591 Patients Infected With SARS-CoV-2 Admitted to ICUs of the Lombardy Region, Italy. JAMA 2020;323:1574-81.

7. Grasselli G, Pesenti A, Cecconi M. Critical Care Utilization for the COVID-19 Outbreak in Lombardy, Italy: Early Experience and Forecast During an Emergency Response. JAMA 2020 Epub. doi: 10.1001/jama.2020.4031

8. Italian National Institute of Statistics. [Impatto dell'epidemia covid-19 sulla mortalità totale della popolazione residente primo trimestre 2020]. Available from: https:/www.istat.it/it/files/ 2020/07 /Rapp_Istat_Iss 9luglio.pdf [Website in Italian].

9. Istituto Superiore di Sanità. [Sorveglianza integrata COVID19 in Italia. 2020]. Available from: https://www.epicentro.iss. it/coronavirus/sars-cov-2-dashboard [Website in Italian].

10. Rhodes A, Ferdinande P, Flaatten H, Guidet B, Metnitz PG, Moreno RP. The variability of critical care bed numbers in Europe. Intensive Care Med 2012;38:1647-53.

11. Regione Toscana. [Linee di indirizzo per la gestione del percorso COVID-19 in ambito ospedaliero e peri-ospedaliero.] 2020. Available from: http://www301.regione.toscana.it/bancadati/atti/Contenuto.xml?id $=5247300 \&$ nomeFile $=$ Ordinanza _del_Presidente_n.14_del_17-03-2020-Allegato-A. [Website in Italian].

12. Rosenbaum L. Facing Covid-19 in Italy - Ethics, Logistics, and Therapeutics on the Epidemic's Front Line. N Engl J Med 2020;382:1873-5.

13. Kokkinakis I, Selby K, Favrat B, Genton B, Cornuz J. [Covid19 diagnosis : clinical recommendations and performance of nasopharyngeal swab-PCR]. Rev Med Suisse 2020;16:699701. [Article in French].

14. Erika P, Andrea V, Cillis MG, et al. Triage decision-making at the time of COVID-19 infection: the Piacenza strategy. Intern Emerg Med 2020;15:879-82.

15. Gargani L, Soliman-Aboumarie H, Volpicelli G, et al. Why, when, and how to use lung ultrasound during the COVID-19 pandemic: enthusiasm and caution. Eur Heart J Cardiovasc Imaging. 2020; jeaa163. doi: 10.1093/ehjci/jeaa163

16. De Filippo O, D’Ascenzo F, Angelini F, et al. Reduced Rate of Hospital Admissions for ACS during Covid-19 Outbreak in Northern Italy. N Engl J Med 2020;283:88-9.

17. La Nazione. [A Viareggio Passeggiata e negozi strapieni. "L'incoscienza ci ha spaventati".] La Nazione 2020. Available from: https://www.lanazione.it/viareggio/cronaca/versiliacoronavirus-1.5062393. [Article in Italian].

18. Italian Civil Protection Department. [Decreto ministeriale del 13 febbraio 2001: criteri di massima per i soccorsi sanitari nelle catastrofi. 2001.] Available from: http:/www.protezionecivile.gov.it/amministrazione-trasparente/provvedimenti/dettaglio/-/asset_publisher/default/content/decreto-ministerialedel-13-febbraio-2001-criteri-di-massima-per-i-soccorsi-sanitari-nelle-catastrofi. [Website in Italian].

19. Decreto Legislativo 9 Aprile 2008, n. 81. [Testo Unico sulla salute e sicurezza sul lavoro]. 2008. Available from: https://www.lavoro.gov.it/documenti-e-norme/studi-e-statistiche/Documents/Testo\%20Unico $\% 20$ sulla $\% 20$ Salute $\% 20 \mathrm{e} \%$ 20Sicurezza\%20sul\%20Lavoro/Testo-Unico-81-08-EdizioneGiugno\%202016.pdf [Article in Italian].

20. Remuzzi A, Remuzzi G. COVID-19 and Italy: what next? Lancet 2020;395:1225-8. 